\title{
ON THE SYMMETRIES OF LORENTZIAN FOUR-DIMENSIONAL GENERALIZED SYMMETRIC SPACES OF TYPE C
}

\author{
LAKEHAL BELARBI
}

\begin{abstract}
We consider the four-dimensional generalized symmetric spaces of type C, equipped with a left-invariant Lorentzian metric. We completely describe its affine, homothetic and Killing vector fields. We also obtain a full classification of its Ricci, curvature and matter collineations.
\end{abstract}

MSC 2010. 53C50, 53B30.

Key words. Generalized symmetric spaces, left-invariant metrics, Killing vector fields, affine vector fields, Lorentzian metrics, curvature and matter collineations.

\section{REFERENCES}

[1] W. Batat, M. Brozos-Vázquez, E. García-Río, and S. Gavino-Fernández, Ricci solitons on Lorentzian manifolds with large isometry groups, Bull. Lond. Math. Soc., 43 (2011), 1219-1227.

[2] W. Batat and K. Onda, Four-dimensional pseudo-Riemannian generalized symmetric spaces which are algebraic Ricci solitons, Results Math., 64 (2013), 254-267.

[3] W. Batat and K. Onda, Ricci and Yamabe solitons on second-order symmetric, and plane wave 4-dimensional Lorentzian manifolds, J. Geom., 105 (2014), 561-575.

[4] L. Belarbi, On the symmetries of the $\mathrm{Sol}_{3}$ Lie group, accepted in J. Korean Math. Soc., DOI: 10.4134/JKMS.j190198, 1-15.

[5] A. Bouharis and B. Djebbar, Ricci solitons on Lorentzian four-dimensional generalized symmetric spaces, Zh. Mat. Fiz. Anal. Geom., 14 (2018), 132-140.

[6] M. Božek, Existence of generalized symmetric Riemannian spaces with solvable isometry group, Časopis Pro Pěstování Matematiky, 105 (1980), 4, 368-384.

[7] M. Brozos-Vázquez, G. Calvaruso, E. García-Río, and S. Gavino-Fernández, Threedimensional Lorentzian homogeneous Ricci solitons, Israel J. Math., 188 (2012), 385403.

[8] G. Calvaruso, O. Kowalski and A. Marinosci, Homogeneous geodesics in solvable Lie groups, Acta. Math. Hungar., 101 (2003), 4, 313-322.

[9] G. Calvaruso and A. Zaeim, Invariant symmetries on non-reductive homogeneous pseudo-Riemannian fourmanifolds, Rev. Mat. Complut., 28 (2015), 599-622.

[10] G. Calvaruso and A. Zaeim, On the symmetries of the Lorentzian oscillator group, Collect. Math., 68 (2017), 51-67.

The authors would like to thank the referee for valuable suggestions regarding both the contents and exposition of this article.

DOI: $10.24193 /$ mathcluj.2020.2.03 
[11] G. Calvaruso, A. Zaeim, Symmetries of Lorentzian three-manifolds with recurrent curvature, SIGMA Symmetry Integrability Geom. Methods Appl., 12 (2016), 1-12.

[12] E. Calvino-Louzao, J. Seoane-Bascoy, M.E. Vazquez-Abal and R. Vazquez-Lorenzo, Invariant Ricci collineations on three-dimensional Lie groups, J. Geom. Phys., 96 (2015), 59-71.

[13] U. Camci, I. Hussain and Y. Kucukakca, Curvature and Weyl collineations of Bianchi type $V$ spacetimes, J. Geom. Phys., 59 (2009), 1476-1484.

[14] U. Camci and M. Sharif, Matter collineations of spacetime homogeneous Gödel-type metrics, Classical Quantum Gravity, 20 (2003), 2169-2179.

[15] J. Carot, J. da Costa and E.G.L.R. Vaz, Matter collineations: the inverse "symmetry inheritance" problem, J. Math. Phys., 35 (1994), 4832-4838.

[16] G. Hall, Symmetries of curvature structure in general relativity, World Science Lecture Notes in Physics, 2004.

[17] O. Kowalski, Generalized symmetric spaces, Lectures Notes in Math., Springer-Verlag, Berlin, Heidelberg, New York, 1980.

[18] A. Mostefaoui, L. Belarbi and W. Batat, Ricci solitons of five-dimensional Solvable Lie group, PanAmerican Math. J., 29 (2019), 1, 1-16.

[19] G. Shabbir, Proper affine vector fields in spherically symmetic static space-times, Differ. Geom. Dyn. Syst., 8 (2006), 244-252.

Received April 16, 2019

Accepted December 4, 2019
University of Mostaganem (U.M.A.B.)

Laboratory of Pure and Applied Mathematics

Department of Mathematics

B.P.227,27000, Mostaganem, Algeria

E-mail: lakehalbelarbi@gmail.com 